\title{
Bounds and Methods for $k$-Planar Crossing Numbers
}

\author{
Farhad Shahrokhi ${ }^{1 \star}$, Ondrej Sýkora ${ }^{2 \star \star}$, Laszlo A. Székely ${ }^{3 \star \star \star}$ and \\ Imrich Vrt'o ${ }^{4, \dagger}$ \\ 1 Department of Computer Science, University of North Texas \\ P.O. Box 13886, Denton, TX, 76203-3886, USA \\ 2 Department of Computer Science, Loughborough University \\ Loughborough, Leicestershire LE11 3TU, The United Kingdom \\ 3 Department of Mathematics, University of South Carolina \\ Columbia, SC 29208, USA \\ 4 Department of Informatics, Institute of Mathematics \\ Slovak Academy of Sciences \\ Dúbravská 9, 84104 Bratislava, Slovak Republic
}

\begin{abstract}
The $k$-planar crossing number of a graph is the minimum number of crossings of its edges over all possible drawings of the graph in $k$ planes. We propose algorithms and methods for $k$-planar drawings of general graphs together with lower bound techniques. We give exact results for the $k$-planar crossing number of $K_{2 k+1, q}$, for $k \geq 2$. We prove tight bounds for complete graphs.
\end{abstract}

\section{Introduction}

Let $\operatorname{cr}(G)$ denote the standard crossing number of a graph $G$, i.e. the minimum number of crossings of its edges over all possible drawings of $G$ in the plane. For $k \geq 2$, define the $k$-planar crossing number as

$$
\operatorname{cr}_{k}(G)=\min \left\{\operatorname{cr}\left(G_{1}\right)+\operatorname{cr}\left(G_{2}\right)+\ldots+\operatorname{cr}\left(G_{k}\right)\right\}
$$

where the minimum is taken over all edge disjoint subgraphs $G_{i}=\left(V, E_{i}\right)$, $i=1,2, \ldots, k$, so that $E=E_{1} \cup E_{2} \cup \ldots \cup E_{k}$.

Motivated by printed circuit boards, Owens [9] introduced the biplanar crossing number of a graph $G$, i.e. the case $k=2$. He described a biplanar drawing of the complete graph $K_{n}$ with $\mathrm{cr}_{2}\left(K_{n}\right) \leq 7 n^{4} / 1536+O\left(n^{3}\right)$. A survey on biplanar crossing numbers is in 5. Determining $\operatorname{cr}_{k}(G)$ has application to the design of multilayer VLSI circuits [1].

* This research was supported by the NSF grant CCR9988525.

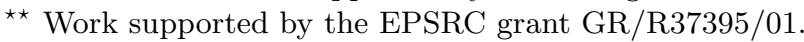

$\star \star \star$ This author was visiting the National Center for Biotechnology Information, NLM, NIH, with the support of the Oak Ridge Institute for Science and Education. This research was partially supported by the NSF contract 0072187.

$\dagger$ Supported partially by the VEGA grant No. 02/3164/23. 
Much of this paper extends ideas of the papers [5] and [12] investigating the biplanar crossing number to the $k$-planar crossing number. Section 2 gives general bounds for the $k$-planar crossing number and exposes an important extremal problem: how does $\mathrm{cr}_{k}(G)$ decrease when $k$ increases?

Section 3 yields unexpected exact results for the $k$-planar crossing number of some complete bipartite graphs. Complete bipartite graphs $K_{p, q}$ are also the best studied graphs with respect to planar crossing numbers. Exact results are known only for $p \leq 6$ and arbitrary $q$, 6]. Crossing numbers of bipartite graphs drawn on surfaces of higher geni were determined only for $p \leq 3$, and arbitrary $q$, 10. Thus our results belong to the same rare class of exact results on crossing numbers (for bipartite graphs), and are direct extensions of the results of [5] for $\mathrm{cr}_{2}\left(K_{5, n}\right)$ and $\mathrm{cr}_{2}\left(K_{6, n}\right)$. We spell out the results in more details. Recall that the thickness $\theta(G)$ of $G$ is the minimum number of planar graphs whose union is $G$. By definition, $\operatorname{cr}_{k}(G)=0$ if and only if $\theta(G) \leq k$. Beineke et al. [4] proved that the thickness of $K_{p, q}$ is given by

$$
\theta\left(K_{p, q}\right)=\left\lceil\frac{p q}{2(p+q-2)}\right\rceil
$$

except, possibly, when $p \leq q$ are both odd and there exists an integer $k$ such that $\frac{1}{4}(p+5) \leq k \leq \frac{1}{2}(p-3)$ and $q=\lfloor 2 k(p-2) /(p-2 k)\rfloor$. According to (1) $\operatorname{cr}_{k}\left(K_{2 k, q}\right)=0$, for $k \geq 2$ and any $q$, so the first interesting bipartite graph is $K_{2 k+1, q}$. We prove that for $k \geq 2, q \geq 1$

$$
\operatorname{cr}_{k}\left(K_{2 k+1, q}\right)=\left\lfloor\frac{q}{2 k(2 k-1)}\right\rfloor\left(q-k(2 k-1)\left\lfloor\frac{q}{2 k(2 k-1)}\right\rfloor-k(2 k-1)\right)
$$

and for $k \geq 2$, and $1 \leq q \leq 4 k^{2}$

$$
\operatorname{cr}_{k}\left(K_{2 k+2, q}\right)=2\left\lfloor\frac{q}{2 k^{2}}\right\rfloor\left(q-k^{2}\left\lfloor\frac{q}{2 k^{2}}\right\rfloor-k^{2}\right) .
$$

Section 4 improves on the general bounds for the $k$-planar crossing numbers of complete and complete bipartite graphs. The improvement means constant multiplicative factors.

\section{General Bounds}

Little is known about lower bounds for the $k$-planar crossing number in general. Some of the lower bounds for crossing numbers, mutatis mutandis apply to $k$ planar crossing numbers. For example, if $G=(V, E),|V|=n,|E|=m$, then the lower bound resulting from Euler's formula, $\operatorname{cr}(G) \geq m-3 n+6$ for $n \geq 3$, generalizes to

$$
\mathrm{cr}_{k}(G) \geq m-k(3 n-6) .
$$

There is a strengthening of the lower bound resulting from Euler's formula for graphs $G$ with girth $g, \operatorname{cr}(G) \geq m-g(n-2) /(g-2)$ for $n \geq g$; and we get

$$
\mathrm{cr}_{k}(G) \geq m-\frac{g k}{g-2}(n-2) \text {. }
$$


We state a $k$-planar version of Leighton's Lemma 7] for crossing numbers (note that we do not go for the best constants here, since the best constant is always getting improved even for the ordinary crossing number).

Lemma 1. For a simple graph $G$ with $n$ vertices and $m$ edges, we have $m \leq 6 k n$, or

$$
\operatorname{cr}_{k}(G) \geq \frac{1}{64} \cdot \frac{m^{3}}{n^{2} k^{2}}
$$

Proof. Recall Leighton's Lemma for the ordinary crossing number: $m \leq 4 n$ or $\operatorname{cr}(G) \geq m^{3} / 64 n^{2}$. Consider an optimal $k$-planar drawing of $G$, such that $G_{i}$ is the subgraph drawn on the $i^{\text {th }}$ plane. Assume that the first $x$ graphs have at most $4 n$ edges, while the last $k-x$ graphs have more. We have

$$
\begin{aligned}
\operatorname{cr}_{k}(G) & \geq \sum_{i=x+1}^{k} \operatorname{cr}\left(G_{i}\right) \geq \sum_{i=x+1}^{k} \frac{m_{i}^{3}}{64 n^{2}} \geq \\
& \geq \frac{k-x}{64 n^{2}} \cdot\left(\frac{\sum_{i=x+1}^{k} m_{i}}{k-x}\right)^{3} \geq \frac{1}{64 n^{2}} \cdot \frac{(m-4 n x)^{3}}{(k-x)^{2}} \geq \frac{1}{64} \cdot \frac{m^{3}}{n^{2} k^{2}},
\end{aligned}
$$

where the last inequality holds for $m \geq 6 k n$ according to the sign of the derivative.

Recall that $a(G)$, or arboricity of $G$, is the minimum number of acyclic subgraphs whose union covers $E$. By a well known theorem of Nash-Williams [8]

$$
a(G)=\max _{H \subseteq G}\left\lceil\frac{m(H)}{n(H)-1)}\right\rceil
$$

where the maximum is taken over all subgraphs $H$ of $G$, with $m(H)$ edges and $n(H)$ vertices. It is easily seen that $a(G) \geq \theta(G)$, moreover, $\theta(G) \geq\lceil a(G) / 3\rceil$, since $m(H) \leq 3 n-6$ for any planar graph.

Let $P=\left\{V_{1}, V_{2}, \ldots V_{t}\right\}$ be a partition of $V$. We denote by $E_{i j}$ the set of edges with one end point in $V_{i}$ and the other in $V_{j}$, hence $E_{i i}$ denotes the set of all edges with both end points in $V_{i}$, for $1 \leq i \leq t$. Let $H$ denote the $t$ vertex graph that is obtained by contracting all vertices in $V_{i}$ into one single vertex and removing the multiple edges. We call $H$ the mate of $G$ with respect to $P$, or simply the mate of $G$. Let $T_{1}, T_{2}, \ldots T_{a(H)}$, be a decomposition of the edge set of $H$ into acyclic subgraphs of $H$. Let $d_{i}(x)$ denote the degree of $x \in V(H)$ in $T_{i}, i=1,2, \ldots k$.

Theorem 1. Let $G=(V, E)$, and let $k$ be a given integer. Let $\left\{V_{1}, V_{2}, \ldots V_{t}\right\}$ be a partition of $V$ and let $H=(V(H), E(H))$ denote the mate of $G$. If $k \geq a(H)$, then we can construct in polynomial time a $k$-planar drawing of $G$ with at most

$$
t p^{2}+2 p q|E(H)|+\sum_{i=1}^{k} p^{2} \sum_{x \in V(H)} d_{i}^{2}(x)
$$

crossings, where $p=\max \left\{\left|E_{i i}\right|\right\}$ and $q=\max \left\{\left|E_{i j}\right|\right\}, i, j=1,2, \ldots t$. 
Proof Sketch. Consider a drawing of each $T_{i}, i=1,2, \ldots, k$ in plane $i$, with no crossings, so that the vertices are placed in the corners of a convex polygon, and each edge is drawn using one straight line segment. Now, replace each vertex $j \in V(H)$ with the set $V_{j}$. In particular, place the vertices of $V_{j}$ in a very small neighborhood around $j$. Next, draw the edges in $E$ with straight line segments using the drawings of $T_{i}$ 's, to produce a $k$-planar drawing of $G$. There will be 3 kinds of crossings:

(a) between edges of $E_{i i}$,

(b) between edges of $E_{i i}$, and edges of $E_{i j}, i \neq j$, and finally

(c) between edges $E_{i j}$, where $i, j=1,2, \ldots k$ and $i \neq j$.

The terms in the theorem correspond to these 3 cases. Note that the estimate for (b) is $p q \sum_{i=1}^{k} \sum_{x \in V(H)} d_{i}(x)=2 p q|E(H)|$.

Theorem 1 can be used effectively, if the degrees appearing in the last term are small. In fact, in certain cases one can decompose $G$ into a number of (cyclic) outer planar graphs of small maximum degree, and still use the method of Theorem 1 to obtain upper bounds for $\mathrm{cr}_{k}(G)$. In this paper, we have obtained exact values of $\operatorname{cr}_{k}(G)$ for certain graphs in this way. Nonetheless, the acyclic decompositions into forests of small maximum degree has been also studied. Let $a_{d}(G)$ denote the degree bounded arboricity, that is the minimum number of forests that the edges of $G$ can be decomposed to so that the maximum degree of each forest is bounded by $d$. Truszczýnski 14] conjectured that for every multigraph $G$ and $d \geq 2$,

$$
a_{d}(G)=\left\{\begin{array}{l}
\Delta(G) / d \text { or } 1+\Delta(G) / d \text { if } a(G)=\Delta(G) / d, \\
\max (a(G),\lceil\Delta(G) / d\rceil) \text { otherwise. }
\end{array}\right.
$$

Truszczýnski actually proved his conjecture for complete and complete bipartite graphs, and also for the case $d \geq \Delta(G)+1-a(G)$. Combining Theorem 1 with (4), we immediately obtain

Corollary 1. For $n \geq 1 \mathrm{cr}_{k}\left(K_{n}\right)=O\left(n^{4} / k^{2}\right)$.

However, Corollary 1 1 also follows from the next theorem:

Theorem 2. For any graph $G$ on $n$ vertices and $m$ edges,

$$
\operatorname{cr}_{k}(G) \leq \frac{1}{12 k^{2}}\left(1-\frac{1}{4 k}\right) m^{2}+O\left(\frac{m^{2}}{k n}\right) .
$$

The corresponding drawing can be found in polynomial time. For any graph $G$,

$$
\operatorname{cr}_{k}(G) \leq \frac{2 \operatorname{cr}(G)}{k^{\log _{2} \frac{8}{3}}}=\frac{2 \operatorname{cr}(G)}{k^{1.4708 \ldots}} .
$$

Proof. The first upper bound follows from our paper [11] (Corollary 3.2) and a simple observation that a drawing of a graph $G$ in $2 k$ pages gives a drawing of the graph $G$ in $k$ planes. The second upper bound follows by iteration from the inequality $\mathrm{cr}_{2}(G) \leq \frac{3}{8} \mathrm{cr}(G)$, proved in [5].

One challenging question is how $\mathrm{cr}_{k}$ changes from $\operatorname{cr}(G)$ to 0 , as $k$ increases from 1 to the thickness of $G, \theta(G)$. 


\section{Exact Results}

Theorem 3. For $k \geq 2, q \geq 1$

$$
\operatorname{cr}_{k}\left(K_{2 k+1, q}\right)=\left\lfloor\frac{q}{2 k(2 k-1)}\right\rfloor\left(q-k(2 k-1)\left(\left\lfloor\frac{q}{2 k(2 k-1)}\right\rfloor+1\right)\right) .
$$

Proof. Upper bound. Beineke [2] proved that the thickness of $K_{2 k+1,2 k(2 k-1)}$ is $k$ by describing a drawing of $K_{2 k+1,2 k(2 k-1)}$ in $k$ planes without crossings. We extend this drawing to a drawing of $K_{2 k+1, q}$ in $k$ planes with minimum number of crossings. Let $u_{1}, u_{2}, \ldots, u_{2 k+1}$ be the vertices of the first partition. Let $v_{1}, v_{2}, \ldots, v_{2 k(2 k-1)}$ be the vertices of the second partition. Beineke's drawing possesses the following properties.

1. On every plane, all $v_{i}$ 's lie on the vertices of the regular $2 k(2 k-1)$-gon.

2. All $u_{j}$ 's lie inside or outside of the polygon.

3. The edges do not cross.

4. For every $v_{i}$, its degree on exactly one plane is 3 and 2 on the remaining $(k-1)$ planes. Moreover, on every plane, the vertex $v_{i}$ has a neighbor inside and a neighbor outside the polygon.

Fig. 1 shows the case $k=3$, i.e. a drawing of $K_{7,30}$ in 3 planes without crossings. The graphs on the same row are drawn on the same plane. The left (right) graph corresponds to the inside (outside) part of the drawing on a plane.

Now consider $K_{2 k+1, q}$ and assume that $q=2 k(2 k-1) a+b$, where $a, b$ are integers and $0 \leq b<2 k(2 k-1)$. Partition the $q$-vertices into $2 k(2 k-1)$ almost equal sets $S_{1}, S_{2}, \ldots, S_{2 k(2 k-1)}$, where $2 k(2 k-1)-b$ sets have $a$ vertices, and $b$ sets have $a+1$ elements. On every plane, replace each vertex $v_{j}$ by the set $S_{j}$ such that its vertices lie on a very short arc and the arcs do not interfere. Join every vertex $S_{i}$ to all vertices of $S_{j}$ on a plane iff $u_{i}$ was adjacent to $v_{j}$ on that plane in the Beineke's drawing. Clearly the total number of crossings is

$$
\sum_{j=1}^{2 k(2 k-1)}\left(\begin{array}{c}
\left|S_{j}\right| \\
2
\end{array}\right)
$$

The above sum turns to

$$
b\left(\begin{array}{c}
a+1 \\
2
\end{array}\right)+(2 k(2 k-1)-b)\left(\begin{array}{l}
a \\
2
\end{array}\right)=a(b+k(2 k-1)(a-1)),
$$

which gives the claimed value by substituting $a=\lfloor q /(2 k(2 k-1))\rfloor$ and $b=$ $q-2 k(2 k-1) a$.

Lower bound. We will proceed by induction on $q$. The claim is obviously true for $q \leq 2 k(2 k-1)$. The claim is also true for $2 k(2 k-1) \leq q \leq 4 k(2 k-1)$ as the RHS of (5) equals $q-2 k(2 k-1)$, which is a lower bound given by (2). Hence assume that the claim is true for some $q \geq 4 k(2 k-1)$. Using the counting argument with $H=K_{2 k+1, q}, G=K_{2 k+1, q+1}$, i.e. counting the number of crossings 

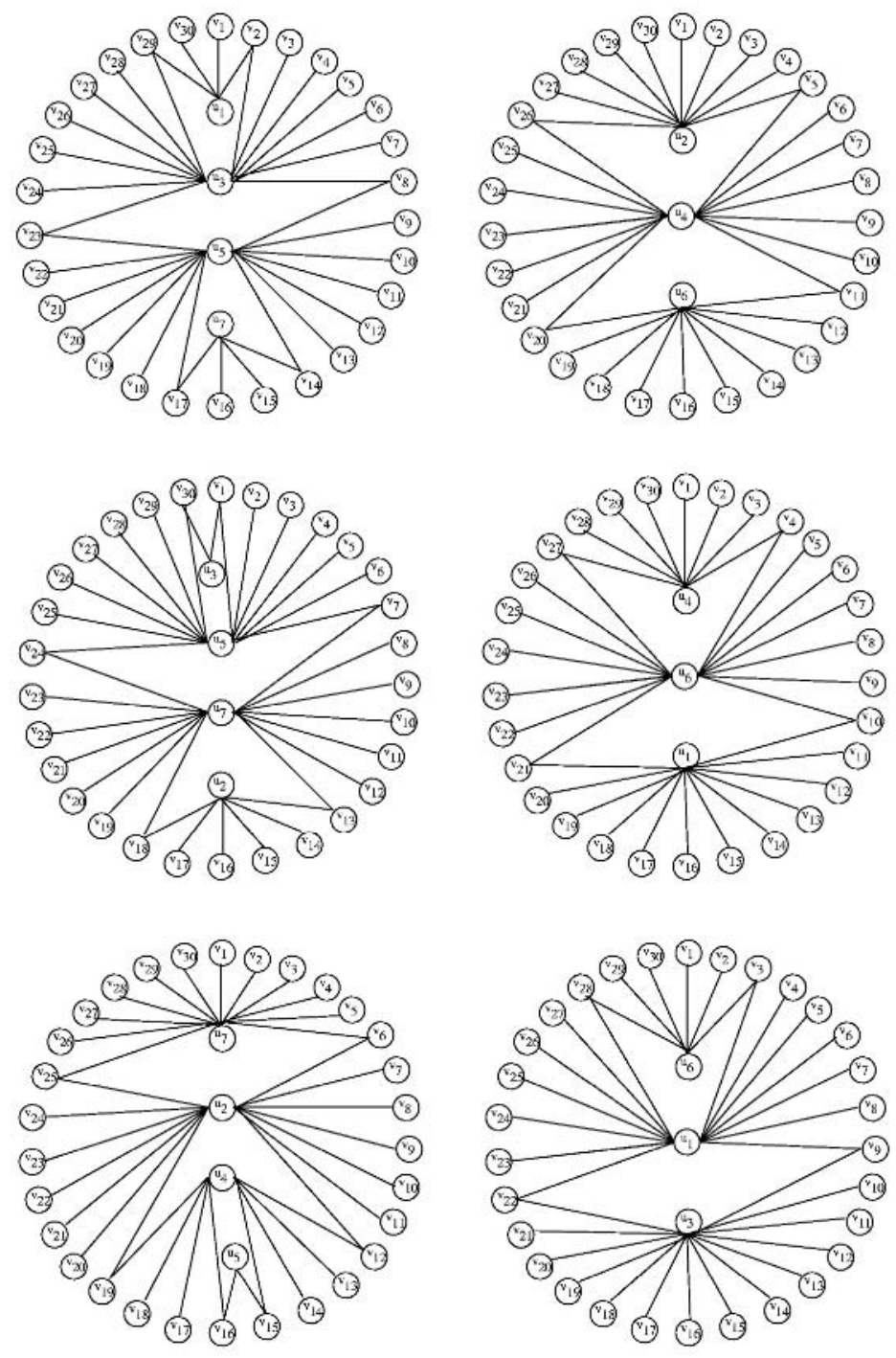

Fig. 1. A drawing of $K_{7,30}$ in 3 planes without crossings.

produced by all occurrencies of $H$ in $G$ and dividing it by the multiplicity of each crossing, we have

$$
\operatorname{cr}_{k}\left(K_{2 k+1, q+1}\right)-\left\lfloor\frac{q+1}{2 k(2 k-1)}\right\rfloor\left(q+1-k(2 k-1)\left(\left\lfloor\frac{q}{2 k(2 k-1)}\right\rfloor+1\right)\right)
$$




$$
\begin{aligned}
\geq\left\lceil\frac{\left(\begin{array}{c}
q+1 \\
q
\end{array}\right)}{\left(\begin{array}{c}
q-1 \\
q-2
\end{array}\right)} \operatorname{cr}_{k}\left(K_{2 k+1, q}\right)\right]-\left\lfloor\frac{q+1}{2 k(2 k-1)}\right\rfloor\left(q+1-k(2 k-1)\left(\left\lfloor\frac{q}{2 k(2 k-1)}\right\rfloor+1\right)\right) \\
\geq \\
\geq\left\lceil\frac{q+1}{q-1}\left\lfloor\frac{q}{2 k(2 k-1)}\right\rfloor\left(q-k(2 k-1)\left(\left\lfloor\frac{q}{2 k(2 k-1)}\right\rfloor+1\right)\right)\right. \\
\left.-\left\lfloor\frac{q+1}{2 k(2 k-1)}\right\rfloor\left(q+1-k(2 k-1)\left(\left\lfloor\frac{q}{2 k(2 k-1)}\right\rfloor+1\right)\right)\right] .
\end{aligned}
$$

To conclude the proof, it is sufficient to show that for $q \geq 4 k(2 k-1)$ the expression inside the big brackets of the last line is greater than -1 . Let $q=$ $2 k(2 k-1) a+b$, as above. Distinguish two cases.

If $b<2 k(2 k-1)-1$ then the expression inside the big brackets equals

$$
\frac{q+1}{q-1} a(q-k(2 k-1)(a+1))-a(q+1-k(2 k-1)(a+1))=\frac{-a-b}{q-1}>-1 .
$$

If $b=2 k(2 k-1)-1$ then the expression inside the big brackets equals

$$
\frac{q+1}{q-1} a(q-k(2 k-1)(a+1))-(a+1)(q+1-k(2 k-1)(a+2))=0 .
$$

Theorem 4. For $k \geq 2$

$$
\operatorname{cr}_{k}\left(K_{2 k+2, q}\right) \leq 2\left\lfloor\frac{q}{2 k^{2}}\right\rfloor\left(q-k^{2}\left\lfloor\frac{q}{2 k^{2}}\right\rfloor-k^{2}\right) .
$$

The equality holds for $1 \leq q \leq 4 k^{2}$.

Proof. Upper bound. We start with a drawing of $K_{2 k+2,2 k^{2}}$ in $k$ planes without crossings and then extend this drawing to a drawing of $K_{2 k+2, q}$. Denote the vertices of the first partition class by $u_{1}, u_{2}, \ldots, u_{k+1}$ and $v_{1}, v_{2}, \ldots, v_{k+1}$. Denote the vertices of the second partition class by $a_{0}, a_{2}, \ldots, a_{k^{2}-1}$ and $b_{0}, b_{1}, \ldots, b_{k^{2}-1}$.

On the first plane, place the vertices $u_{1}, u_{2}, \ldots, u_{k+1}$ (resp. $v_{1}, v_{2}, \ldots, v_{k+1}$ ) on the positive (resp. negative) part of the $x$ axis, in this order from the origin. Place the vertices $a_{0}, a_{1}, \ldots, a_{k^{2}-1}$ (resp. $b_{0}, b_{1}, \ldots, b_{k^{2}-1}$ ) on the positive (resp. negative) part of the $y$ axis, in this order from the origin. Join $u_{i}$ and $v_{i}$ to $a_{(i-1)(k-1)}, \ldots, a_{i k-i}$ and $b_{(i-1)(k-1)}, \ldots, b_{i k-i}$, for all $i$. On the second plane, the positions of $u_{i}$ 's and $v_{i}$ 's remain unchanged. Shift $a_{j}$ 's (resp. $b_{j}$ 's) cyclically up (down) by $k$ position. Join $u_{i}$ and $v_{i}$ to $a_{(i-2)(k-1)}, \ldots, a_{(i-1)(k-1)}$ and $b_{(i-2)(k-1)}, \ldots, b_{(i-1)(k-1)}$, where the indices are computed modulo $k^{2}$. Continuing in this drawing for all planes we get a drawing of $K_{2 k+2,2 k^{2}}$ in $k$ planes without crossings. See Fig. 2 for the case $k=3$.

Now consider $K_{2 k+2, q}$. Partition the $q$ vertices into $2 k^{2}$ almost equal sets, $A_{0}, A_{1}, \ldots, A_{k^{2}-1}$ and $B_{0}, B_{1}, \ldots, B_{k^{2}-1}$. Replace every $a_{j}$ and $b_{j}$ by $A_{j}$ and $B_{j}$ and join $u_{i}$ and $v_{i}$ to $A_{j}$ and $B_{j}$ on a plane iff $u_{i}$ and $v_{i}$ were adjacent to $a_{j}$ and $b_{j}$ on that plane. A simple counting shows that the number of crossings is

$$
\sum_{j=0}^{k^{2}-1} 2\left(\left(\begin{array}{c}
\left|A_{j}\right| \\
2
\end{array}\right)+\left(\begin{array}{c}
\left|B_{j}\right| \\
2
\end{array}\right)\right)
$$




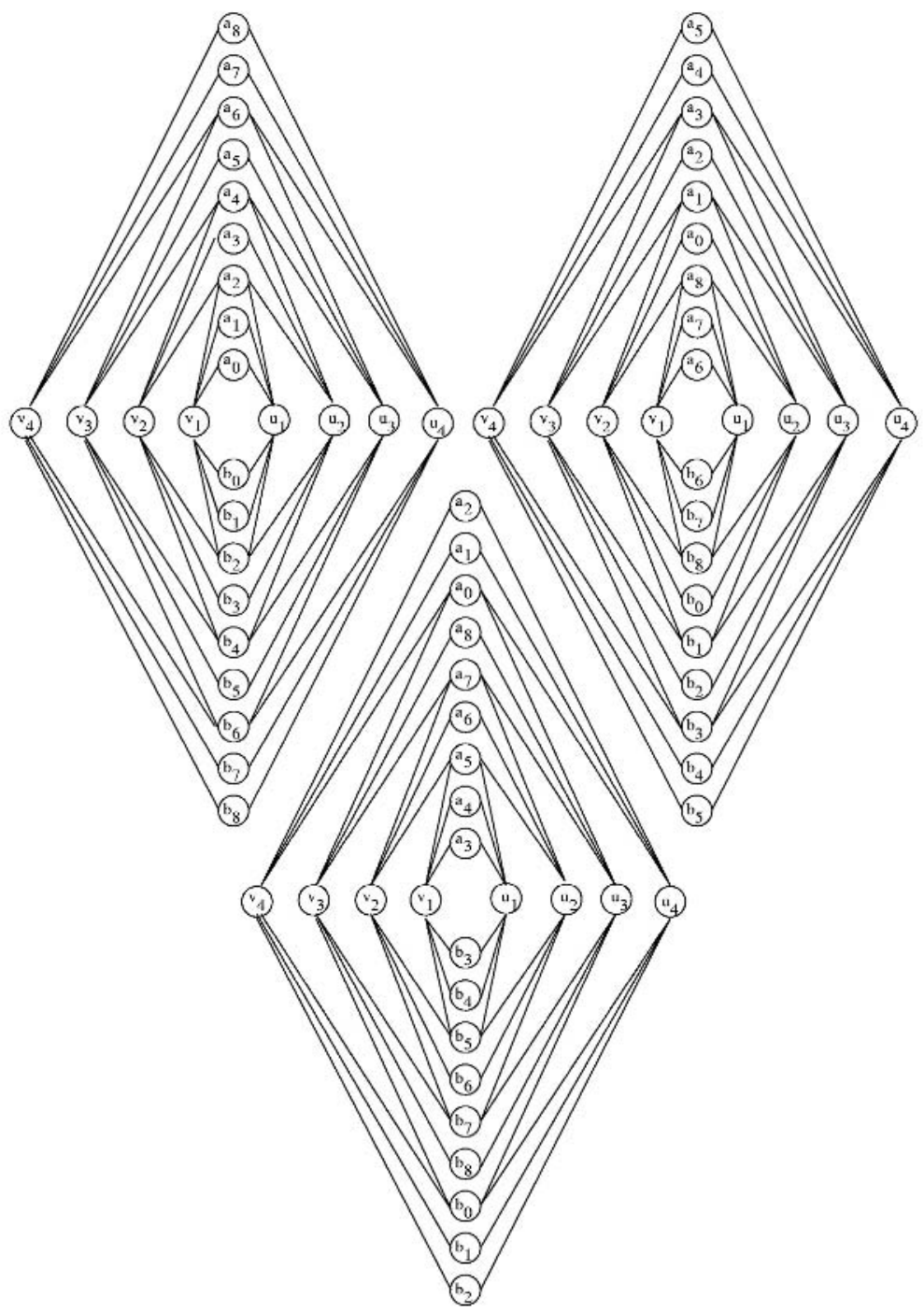

Fig. 2. A drawing of $K_{8,18}$ in 3 planes without crossings.

The rest is similar to the proof of Theorem 3 ,

Lower bound. As $\theta\left(K_{2 k+2,2 k^{2}}\right)=k$, from (1), $\mathrm{cr}_{k}\left(K_{2 k+2, q}\right)=0$, for $q \leq 2 k^{2}$. Assume $2 k^{2} \leq q \leq 4 k^{2}$. In this interval the RHS of (6) equals $2 q-4 k^{2}$, which is the lower bound given by (2). 


\section{Improved Bounds on Complete and Complete Bipartite Graphs}

\subsection{Lower Bounds}

For specific graphs we can strengthen the lower bound by the standard counting argument.

Theorem 5. For $p \geq 6 k-1$ and $q \geq \max \left\{6 k-1,2 k^{2}\right\}$

$$
\operatorname{cr}_{k}\left(K_{p, q}\right) \geq \frac{1}{3(3 k-1)^{2}}\left(\begin{array}{l}
p \\
2
\end{array}\right)\left(\begin{array}{l}
q \\
2
\end{array}\right) \text {. }
$$

Proof. The estimation (2) gives

$$
\operatorname{cr}_{k}\left(K_{6 k-1,6 k-1}\right) \geq 12 k^{2}-4 k+1 .
$$

Using the counting argument with $H=K_{6 k-1,6 k-1}$ and $G=K_{p, q}$ we have

$$
\operatorname{cr}_{k}\left(K_{p, q}\right) \geq \frac{\left(\begin{array}{c}
p \\
6 k-1
\end{array}\right)\left(\begin{array}{c}
q \\
6 k-1
\end{array}\right)}{\left(\begin{array}{c}
p-2 \\
6 k-3
\end{array}\right)\left(\begin{array}{c}
q-2 \\
6 k-3
\end{array}\right)} \operatorname{cr}_{k}\left(K_{6 k-1,6 k-1}\right)>\frac{1}{3(3 k-1)^{2}}\left(\begin{array}{l}
p \\
2
\end{array}\right)\left(\begin{array}{l}
q \\
2
\end{array}\right)
$$

Theorem 6. For $n \geq 2 k^{2}+6 k-1$

$$
\operatorname{cr}_{k}\left(K_{n}\right) \geq \frac{1}{2(3 k-1)^{2}}\left(\begin{array}{l}
n \\
4
\end{array}\right) .
$$

Proof. Let $n=p+q$. Combining the counting argument with $H=K_{p, q}$ and $G=K_{n}$ with the lower bound from Theorem 8 we get the claim.

\subsection{Upper Bounds}

For special values of $k$ we can improve on the upper bounds from Corollary 1 and Theorem 2 .

Theorem 7. Let $k-1$ be a power of a prime. For $n \geq(k-1)^{2}$

$$
\operatorname{cr}_{k}\left(K_{n}\right) \leq \frac{1}{64} \frac{k}{(k-1)^{3}}\left(n+k^{2}\right)^{4} \text {. }
$$

Proof. To appear in the full verion.

For the complete bipartite graphs and arbitrary $k$ we can extend the construction from the Section 3 .

Theorem 8. For $p \geq 2 k+2$ and $q \geq 2 k^{2}$

$$
\operatorname{cr}_{k}\left(K_{p, q}\right) \leq \frac{k^{2}+k+2}{16 k^{2}(k+1)^{2}}(p+2 k+1)^{2}\left(q+2 k^{2}-1\right)^{2} .
$$

Proof. To appear in the full version. 


\section{References}

1. Aggarwal, A., Klawe, M., Shor, P., Multi-layer grid embeddings for VLSI, Algorithmica 6, (1991), 129-151.

2. Beineke, L.W., Complete bipartite graphs: Decomposition into planar subgraphs, Chapter 7, in: A Seminar on Graph Theory, (F. Harary, ed.), Selected Topics in Mathematics, Holt, Rinehart and Winston, New York, 1967, 43-53.

3. Beineke, L.W., Biplanar graphs: A survey, Computers and Mathematics with Applications 34 (1997), 1-8.

4. Beineke, L.W., Harary, F., Moon, J.W., On the thickness of the complete bipartite graphs, Proc. of the Cambridge Philosophical Society 60 (1964), 1-5.

5. Czabarka, É., Sýkora, O., Székely, L.A., Vrto, I., Biplanar crossing numbers: A survey of results and problems, in: Finite and Infinite Combinatorics, (T. Fleiner, G. O. H. Katona, eds.), Bolyai Society Mathematical Studies, Akadémia Kiadó, Budapest, to appear.

6. Kleitman, D.J., The crossing number of $K_{5, n}$, J. Combinatorial Theory 9 (1970), $315-323$.

7. Leighton, T. F., Complexity Issues in VLSI, MIT Press, Cambridge 1983.

8. Nash-Williams, J.A., Edge disjoint spanning trees of finite graphs, J. London Math. Soc., 36 (1961), 445-450.

9. Owens, A., On the biplanar crossing number, IEEE Transactions on Circuit Theory 18 (1971), 277-280.

10. Richter, R.B., Šrán̆, J., The crossing number of $K_{3, n}$ in a surface, J. Graph Theory 21 (1996), 51-54.

11. Shahrokhi, F., Sýkora, O., Székely, L.A., Vrto, I., The book crossing number of graphs, J. Graph Theory 21 (1996), 413-424.

12. Sýkora, O., Székely, L.A., Vrťo, I., Crossing numbers and biplanar crossing numbers: using the probabilistic method, submitted.

13. Shahrokhi, F., Sýkora, O., Székely, L.A., Vrto, I., Shahrokhi, F., Sýkora, O., Székely, L.A., Vrto, I., Bounds for convex crossing numbers, in: Proc. 9th Intl. Computing and Combinatorics Conference, Lecture Notes in Computer Science 2697, Springer Verlag, Berlin 2003, 487-495.

14. Truszczyński, M., Decomposition of graphs into forests with bounded maximum degree, Discrete Mathematics 98 (1991), 207-222.

15. White A.T., Beineke, L.W., Topological graph theory, in: Selected Topics in Graph Theory, (L.W. Beineke, R.J. Wilson, eds.), Academic Press, New York 1978, 15-50. 